\title{
Enhacement of the Viability of Lactobacillus plantarum during the Preservation and Storage Process Based on the Response Surface Methodology
}

\author{
Girme Gisela, Arosemena Esteban Leonardo, Perez Lucia, Vera Rodrigo, Grau Eduard, \\ Calvo M. Angeles \\ Grupo de Microbiología Aplicada y Medio-ambiental, Facultad de Veterinaria, Universidad Autónoma de \\ Barcelona, Bellaterra (Barcelona), Spain \\ Email: gisela.girme@gmail.com
}

Received 18 June 2014; revised 20 July 2014; accepted 5 August 2014

Copyright (C) 2014 by authors and Scientific Research Publishing Inc.

This work is licensed under the Creative Commons Attribution International License (CC BY). http://creativecommons.org/licenses/by/4.0/

(c) (i) Open Access

\section{Abstract}

Objective: The Response Surface Methodology (RSM) is a commonly used system to optimize cell viability of probiotic strains when they are subjected to different preservation and storage processes. Methods and Results: To determine the optimal levels of incorporation of several cry oprotectants (skim milk, sucrose and trehalose) in the freeze-drying process of Lactobacillus plantarum, a range of experiments based on a Rotational Central Composite design (CCD) were conducted. The results were adjusted to a quadratic model, resulting in the presence of interaction between the different variables. Solving a regression equation, we obtained the optimum concentrations of cryoprotective agents: $24.06 \%$ milk powder, $6.22 \%$ sucrose, $5.63 \%$ trehalose. To visualize the interactions between the three variables involved in the study, Design Expert ${ }^{\circledR}$ software was used. Conclusions: The analysis reveals that while trehalose has a direct effect on the viability of $L$. plantarum, skim milk and sucrose exert quadratic effects. There are also interactions between cryoprotectants, which emphasize the synergies produced between milk and sucrose and between sucrose and trehalose, which allows maintaining the viability of $L$. plantarum. Significance and Impact of the Study: The addition of new oligosaccharides as trehalose in premixtures for functional feed can maintain the viability of $L$. plantarum during longer periods of time, ensuring the proper administration of probiotics to their destinations.

\section{Keywords}

Cryoprotectants, Freeze-Drying Process, Probiotics, Response Surface Methodology, Central Composite Design 


\section{Introduction}

According to the definition probiotic is: "live microorgnisms which administered in adequate concentration confer a health benefit” (WHO). Numerous studies have conducted on the efficacy of probiotics in improving human health [1]-[4] and more recently in animal health [5]-[7].

Interest in the use of probiotics in animal feed has increased significantly after the prohibition of the use of antibiotics as growth promoters by the European Union (Regulation CEE 1831/2003). The persistent economic pressures to optimize production parameters have made necessary the search of new strategies for improvement, including also the use of prebiotics, enzymes, organic acids and immune stimulants [8], which entered into the diet of animals as functional foods.

A functional food is one in which the introduction of one or more new ingredients allows the product acquires a new function, often related to improving health or preventing disease [9]. Functional foods are one of the most promising growth areas in the food industry. These foods are the result of combining the basic ingredients of the mixture with other ingredients that add value. The new ingredients are formulated as premix prior to then be placed in the base mixture, resulting in a nutritious custom product [10].

Producing effective nutritional premixes can become a difficult task. The two main challenges in the design and production of a premix are a good consistency and a high stability, ensuring its maintenance during its lifetime. Homogeneity and stability of premix affect not only the quality but also the finished products. When the addition of the additive should be carried out only in small amounts, such as probiotics, it helps dissolve the ingredients before adding them to the mix to help correct prior distribution premix [10].

Lactic acid bacteria (LAB) represent a major group of microorganisms used as starter cultures and probiotics in the food industry. The industrial application of LAB depends on concentration and conservation technologies that are required to ensure the long term stability of cultures in terms of viability and functional activity [11] [12]. It is essential, both technologically and economically, maximizing the viability of laboratory cultures during drying and subsequent storage for long periods [13] [14].

There are several mechanisms that allow us to preserve the viability of the bacteria over time, being cryopreservation and lyophilization the most prominent [15] [16].

In the production of compound feed, LAB are subjected to various stressful technological procedures. It is essential to maintain the viability of probiotics to remain effective. Residents of natural intestine, LAB not form spores, once subjected to drying they are unprotected against chemical or physical damage occurring during the granulation process. This makes its use restricted to certain types of formulations that impose low pressure techniques on microorganisms, and is necessary to protect them against the specific heat and mechanical impact during manufacture, transport and storage of food [17].

In addition, the freeze-drying and subsequent storage produce cell viability decrease due to the drying exposes cells to an additional stage of stress processing. Different species show different degrees of survival to freezedrying [18]. The degree of viability loss depends on inherent microorganism factors (strain properties, growing conditions and the state of growth) and other factors inherent in the process (technical parameters such as cooling rate and temperature, the presence of cryoprotectants, and the type of rehydration buffer) [19]. These factors may cause osmotic shock and membrane injury during recrystallization by the formation of intracellular crystals [15] [20].

To minimize this damage, cryoprotectant substances are commonly used. Some sugars are recognized as protectors and used for the preparation of freeze-dried cultures. These sugars stabilize the cell membrane by a mechanism of replacement of water and a series of interactions between membrane phospholipids and sugars [21].

Skim milk and sucrose are commonly used as cryoprotectants [22]. Skimmed milk is considered to be able to prevent cell damage by stabilizing the cell membrane, providing a protective layer for the cells [14], while the protective activity of sucrose suggests that is due to its ability to prevent harmful eutectic fluid cell freezing [22].

Other polysaccharides such as maltose, lactose and trehalose, as well as maltodextrin also have use as cryoprotectants, increasing the viability of lactic acid bacteria during freezing and freeze-drying processes [23]. When the water content of the samples is low, the sugars form a glassy matrix that is characterized by high viscosity and low mobility [20].

Under ideal conditions for drying and storage, trehalose is probably more effective than other oligosaccharides in the preservation of biomaterials [22]. This increased tolerance to desiccation appears to be result of the ability of certain disaccharides to lower the temperature of the membrane during the transition to the drying and 
maintain the structure of the proteins in the dried state [23].

The aim of this study was to evaluate the effect of different cryoprotectants (skim milk powder, sucrose and trehalose) on the viability of a Lactobacillus plantarum probiotic strain when it is subjected to a stressful process of freezing and subsequent lyophilization, determining the optimal combination of cryoprotectants to improve the viability of the preparations before its incorporation into a premix feed.

\section{Materials and Methods}

\subsection{Bacterial Culture and Bacterial Culture Medium}

The organism chosen to perform the study was a strain of Lactobacillus plantarum CECT 8150, isolated from free-living snails and identified by the commercial kit API 50 CHL (Biomerieux ${ }^{\circledR}$ sa, Marcy-Étoile, France) and testing by sequencing PCR (PCR kit MicroSeq ${ }^{\circledR} 500$ 16S rDNA microbial identification, Life TechnologiesApplied Biosystems, Madrid).The microorganism remained viable in the medium Man Rogosa Sharpe (MRS Agar ISO 15214, Ref: 610024, Liofilchem srl, Italy).

\subsection{Cryoprotective Medium and Preparation of Suspension}

The cryoprotectants that were used in this study were skim milk powder (Ref: 06-019-500, Scharlab, Barcelona, Spain), sucrose (PA-ACS Saccharose Ref: 131621. 1211, Panreac Química SA, Barcelona, Spain) and trehalose (D (+)-Trehalose dihydrate BioChemica Ref: A1742, 0025, AppliChem, Darmstadt, Germany). These cryoprotectants are relatively cheap, safe to use and commonly used in the food industry (Khoramnia 2011), these qualities allow the incorporation of probiotics properly protected large quantities of feed, favoring large-scale production.

The range of concentrations used in this study cover the concentrations commonly applied including values that have been previously used in other studies [24] [25]. To obtain the concentrations of each cryoprotectant used to prepare each suspension media, a Central Composite Rotational Design was used (CCD) [24] [25]. The variables used in the experimental design in coded levels (xi) and current levels (Xi) are shown in Table 1. The concept current level refers to the real values unencoded in percentage arising of each variable in the experiment.

To develop the regression equation, the three factors to be tested are encoded according to the following equation:

$$
x i=\frac{\left(X_{i}-X_{o}\right)}{\Delta X_{i} i}=1,2,3, \ldots, k,
$$

where $x$ is the raw value of the independent variable dimensions, $X_{i}$ is the actual value of the independent variable in the central point and $\Delta X i$ is the step change value. Solving this equation gives us the values of the variables at each coded level as shown in Table 1.

To analyze the experimental results, we used Response Surface Methodology (RSM). We tried to adapt the results to a quadratic model to correlate the variability of responses to the independent variables of the study. The system behavior can be explained by the following polynomial quadratic equation:

$$
Y=\beta_{0}+\sum \beta_{i} x_{i}+\sum \beta_{i i} x_{i}^{2}+\sum \beta_{i j} x_{i} x_{j}
$$

where $Y$ is the predicted response, $\beta_{0}$ is the intercept term, $\beta_{i}$ is the linear term and quadratic term is $\beta_{i i}$.

Table 1. Coded levels and the variables used in the CCD.

\begin{tabular}{cccccccc}
\hline Cryoprotectant & \multicolumn{2}{c}{ Symbol } & \multicolumn{2}{c}{ Variable values at each level of each variable and code } \\
& Unencoded & Coded & $-\mathbf{- 1 . 6 8 2}$ & -1 & $\mathbf{0}$ & $+\mathbf{1}$ & $+\mathbf{1 . 6 8 2}$ \\
\hline Skim milk & $X_{1}$ & $x_{1}$ & $5 \%$ & $9.06 \%$ & $15 \%$ & $24.06 \%$ & $25 \%$ \\
Sucrose & $X_{2}$ & $x_{2}$ & $1 \%$ & $2.22 \%$ & $4 \%$ & $6.22 \%$ & $7 \%$ \\
Trehalose & $X_{3}$ & $x_{3}$ & $0 \%$ & $1.62 \%$ & $4 \%$ & $5.63 \%$ & $8 \%$ \\
\hline
\end{tabular}


In the CCD, the total number of combinations to do was $2^{\mathrm{k}}+2 \mathrm{~K}+\mathrm{n}_{0}$, where $\mathrm{K}$ is the number of independent variables and $\mathrm{n}_{0}$ is the number of repetitions of the experiment at the center point, which told us that our case, with 3 variables, we required a total of 20 experiments, which were carried out in duplicate. To perform regression analysis and graphical experiment was used Design Expert ${ }^{\circledR}$ software (version 8.0.7.1, Stat-Ease. Inc, Minneapolis, MN, USA).

Twenty different suspension media were prepared based on the CCD (Table 2). The base solution used for the suspension of cryoprotectants was phosphate buffer saline (PBS) (Phosphate Buffered Saline, pH 7.4, Sigma Aldrich, St. Louis, USA). Ten $\mathrm{mL}$ were prepared for each combination individually and were autoclaved at $110^{\circ} \mathrm{C}$ for 5 minutes.

\subsection{Sample Preparation}

One colony from the MRS agar culture was suspended in MRS broth and was incubated at $37^{\circ} \mathrm{C}$ in a $5 \% \mathrm{CO}_{2}$ atmosphere for 18 hours, at which time the culture was near the stationary phase of growth [26], resulting in a concentration of about $10^{9}$ colony forming units (CFU) $\mathrm{mL}^{-1}$. This was verified by the inoculating $100 \mathrm{~mL}$ of culture on the surface of MRS agar plates and incubating the plates 24 hours $\left(37^{\circ} \mathrm{C}, 5 \% \mathrm{CO}_{2}\right)$.

Two $\mathrm{mL}$ of the inoculum were suspended in $8 \mathrm{~mL}$ of MRS broth and incubated 18 hours $\left(37^{\circ} \mathrm{C}, 5 \% \mathrm{CO} 2\right)$. After this time, the pellet was separated from the liquid portion by centrifugation at 16,000 $\mathrm{g}$ for 5 minutes (RT192, Ortoarlesa, Spain). The pellets were washed twice in $10 \mathrm{~mL}$ of phosphate buffered solution (PBS) by centrifugation. After washing, the pellets were suspend in $10 \mathrm{~mL}$ of different combinations of cryoprotectants previously mentioned (Table 2). PBS was used for negative controls.

\begin{tabular}{cccc}
\hline Table 2. Matrix CCD experimental design. & Sucrose & Trehalose \\
\hline Sample & Skim milk & -1 & -1 \\
\hline $\mathbf{1}$ & -1 & -1 & -1 \\
$\mathbf{2}$ & 1 & 1 & -1 \\
$\mathbf{3}$ & -1 & 1 & -1 \\
$\mathbf{4}$ & 1 & -1 & 1 \\
$\mathbf{5}$ & -1 & -1 & 1 \\
$\mathbf{6}$ & 1 & 1 & 1 \\
$\mathbf{7}$ & -1 & 1 & 1 \\
$\mathbf{8}$ & 1 & 0 & 0 \\
$\mathbf{9}$ & -1.682 & 0 & 0 \\
$\mathbf{1 0}$ & 1.682 & -1.682 & 0 \\
$\mathbf{1 1}$ & 0 & 1.682 & 0 \\
$\mathbf{1 2}$ & 0 & 0 & -1.682 \\
$\mathbf{1 3}$ & 0 & 0 & 1.682 \\
$\mathbf{1 4}$ & 0 & 0 & 0 \\
$\mathbf{1 5}$ & 0 & 0 & 0 \\
$\mathbf{1 6}$ & 0 & 0 & 0 \\
$\mathbf{1 7}$ & 0 & 0 & 0 \\
$\mathbf{1 8}$ & 0 & 0 & 0 \\
$\mathbf{1 9}$ & 0 & 0 & 0 \\
$\mathbf{2 0}$ & 0 & $0 \%$ & 0 \\
$\mathbf{C o n t r o l}$ & $0 \%$ & & 0 \\
\hline & & 0 & 0 \\
\hline
\end{tabular}




\subsection{Lyophilization}

Combinations obtained were prepared to be subjected to lyophilization. Two vials were prepared for each combination, containing $1 \mathrm{~mL}$ of suspension, and were kept at $-20^{\circ} \mathrm{C}$ for $24 \pm 2$ hours. The remaining volume of each suspension were frozen at $-20^{\circ} \mathrm{C}$ for $48 \pm 2$ hours.

The frozen combinations were dehydrated for 24 hours in a freeze-dryer ( -55 LyoQuest Telstar Technologies, Spain), with a collector temperature of $-50^{\circ} \mathrm{C}$ to $5 \times 10^{2}$ mbar. After completing the cycle of lyophilization, the vials were closed in sterile conditions.

\subsection{Determining the Viability of Microorganisms}

\subsubsection{Determination of Viability Pre-Lyophilization}

Once suspended in cryoprotectant media and before being lyophilized, an aliquot of each combination were used to determine the different pre-lyophilization viabilities, which allow the calculation of the subsequent loss of viability. We conducted a dilution stock in Ringer lactate. $100 \mathrm{~mL}$ of appropriate dilutions were seeded on MRS agar plates and were left to incubate at $37^{\circ} \mathrm{C}$ in $5 \% \mathrm{CO}_{2}$ atmosphere for 24 hours for subsequent counting.

\subsubsection{Determining the Conservation of Viability over Time}

To mimic the usual conditions of the premixture preparation, $15 \mathrm{~mL}$ of different cryoprotectants were introduced into sterile and were frozen and lyophilized. As a basis for the premix was employed wheat flour. By their color, allows confirmation of correct homogenization of the mixture at first sight, as well of being a preparation that does not change significantly the composition of the mixture.

Sterile jars were kept for one month at room temperature in a dry place. After this time, each lyophilized jar was granulated with the help of a sterile mortar, and subsequently introduced into a sterile bag with $300 \mathrm{~g}$ of wheat flour. Each mixture was placed in a homogenizer machine (IUL Instruments, Barcelona, Spain) for 10 minutes to ensure proper distribution. Subsequently, the concentration of probiotic was tested by doing a dilution stock from two different aliquots of premix taken from two different areas of each bag, in order to assess the homogeneity of distribution. Dilutions were made with Ringer Lactate and sowing in MRS agar.

\section{Results}

\subsection{Central Composite Rotational Design Analysis Using Response Surface Methodology}

Of all the experimental data that were available to develop the RSM, chose viability in different premixes in CFU/g for the study of the interactions between different cryoprotectants. We considered these results because they allow us to assess both the preservation of viability over time as the homogeneity of the premix. We couldn't asses this last factor if we analyzed other results like the vial viability just after lyophilization or after one month of storage. Using regression analysis on the experimental data, we obtained the table shown below (Table 3).

The adjusted summary that the program offers shows the different models that can be used to analyze the data. For our analysis we chose the quadratic model, which enabled us to visualize how the different terms contributing to the total growing complexity of the model. Once the pattern, he proceeded to carry out their analysis of variance (ANOVA) (Table 4).

Table 3. Prediction of appropriate model to experimental data. The table shows the different types of models that are available. In the last column, the program shows the model that best fits the data entered (suggested) and those models to be discarded.

\begin{tabular}{cccccc}
\hline & \multicolumn{5}{c}{ Adjusted models summary } \\
Models & p-value sequential & Lack of fit p-valor & Adjusted R-squared & Expected R-squared & \\
\hline Lineal & 0.5601 & 0.0001 & -0.0480 & -0.5288 & \\
2FI & 0.0073 & 0.0007 & 0.4726 & 0.3251 & \\
Quadratic & $\underline{0.0032}$ & $\underline{0.0067}$ & $\underline{0.8169}$ & $\underline{0.3023}$ & $\underline{\text { Suggested }}$ \\
Cubic & 0.5438 & 0.0014 & 0.8049 & -11.0894 & Dischargeable \\
\hline
\end{tabular}


Table 4. Analysis of variance summary (ANOVA). The p-value shows that the model is significant. The asterisk values are significant with a p-value $<0.01$.

\begin{tabular}{cccccc}
\hline \multicolumn{5}{c}{ ANOVA for response surface quadratic model } \\
\hline \multicolumn{5}{c}{ Analysis of variance table (sum of squares) } \\
\hline Code & Sum of squares & Mean square & F Value & P-value Prob > F & Significance \\
\hline Model & 2.02 & 0.22 & 10.42 & 0.0005 & significant \\
$\boldsymbol{x}_{\mathbf{1}}$-Skim milk & 0.053 & 0.053 & 2.47 & 0.1471 & \\
$\boldsymbol{x}_{2}$-Sacarose & 0.024 & 0.024 & 1.11 & 0.3165 & \\
$\boldsymbol{x}_{3}$-Trehalose & 0.19 & 0.19 & 8.61 & $0.0149^{*}$ & \\
$\boldsymbol{x}_{\mathbf{1}} \boldsymbol{x}_{2}$ & 0.76 & 0.76 & 35.13 & $0.0001^{*}$ & \\
$\boldsymbol{x}_{\mathbf{1}} \boldsymbol{x}_{3}$ & 0.097 & 0.097 & 4.50 & 0.0600 & \\
$\boldsymbol{x}_{2} \boldsymbol{X}_{3}$ & 0.31 & 0.31 & 14.49 & $0.0034^{*}$ & \\
$\boldsymbol{x}_{\mathbf{1}}{ }^{2}$ & 0.14 & 0.14 & 6.47 & $0.0292^{*}$ & \\
$\boldsymbol{x}_{2}{ }^{2}$ & 0.49 & 0.49 & 22.90 & $0.0007^{*}$ & \\
$\boldsymbol{x}_{3}{ }^{2}$ & 0.027 & 0.027 & 1.27 & 0.2863 & significant \\
Lack of fit & 0.20 & 0.040 & 13.17 & 0.0067 & \\
\hline
\end{tabular}

In Table 4 it can be seen how "F value" model was 10.42, which implied that the model was significant. Only $0.05 \%$ of variability obtained could not be explained by the model. Lack of fit was significant, which indicates that the specified model would not be appropriate for the data.

The terms of the model that had a p-value Prob $>$ F less of 0.05 were significant, in this case $x_{3}, x_{1} x_{2}, x_{2} x_{3}, x_{12}$ and $x_{22}$. Note the effect of trehalose $\left(P x_{3}<0.0007\right)$, which was more significant than the first-order effects of skim milk $\left(P_{X 1}<0.1471\right)$ and sucrose $\left(P_{X 2}<0.3165\right)$. These two components showed no first-order effects alone, but had significant quadratic effects $\left(P x_{1}{ }^{2}<0.0292\right.$ and $\left.P x_{2}{ }^{2}<0.0007\right)$.

Regarding the interactions, the most significant result was obtained by skim milk-sucrose interaction $\left(P x_{1} x_{2}<\right.$ 0.0001). It was also significant trehalose-sucrose influence $\left(P x_{2} X_{3}<0.0034\right)$. The interaction skim milk-trehalose was not significant $\left(P x_{1} x_{3}<0.06\right)$, although the value is close enough to the limit of 0.05 .

For the coefficient of determination $\left(\mathrm{R}^{2}\right)$, we obtained a value of 0.90 . This meant that $10 \%$ of the variability in the response could not be explained by the model. The correlation coefficient (R) was 0.95 , which indicates that there is correlation between predicted and experimental viability.

Finally, multiple regression analysis (Table 5) led us to obtain the quadratic polynomial equation in order to express the cell viability of premix functional L. plantarum:

$$
Y=7.18+0.062 x_{1}+0.042 x_{2}+0.12 x_{3}+0.31 x_{1} x_{2}+0.11 x_{1} x_{3}+0.20 x_{2} x_{3}+0.098 x_{1}^{2}+0.18 x_{2}^{2}+0.044 x_{3}^{2}
$$

where $x_{1}, x_{2}$ and $x_{3}$ are adimensional coded values of the three independent variables of the study, skim milk, sucrose and trehalose, respectively.

\subsection{Effects of Protective Agents}

In order to visualize the interactions between the three variables involved in the study, Design Expert ${ }^{\circledR}$ software allows graphical model that shows functions involving the three factors, keeping one of them at a fixed concentration and varying concentrations of the two remaining elements (3D surface). We obtained three graphs, where each of the variables (in order: skim milk, sucrose and trehalose) were maintained at the concentration corresponding to the value coded 0 (15\%, 5\% and 5\% concentration respectively).

Microbial viability was increased both high concentrations of sucrose and milk and low concentrations of the two cryoprotectants. This response was not seen in the cases where one of the two variables was low and the other at high concentrations. These effects were significant (Figure 1). 


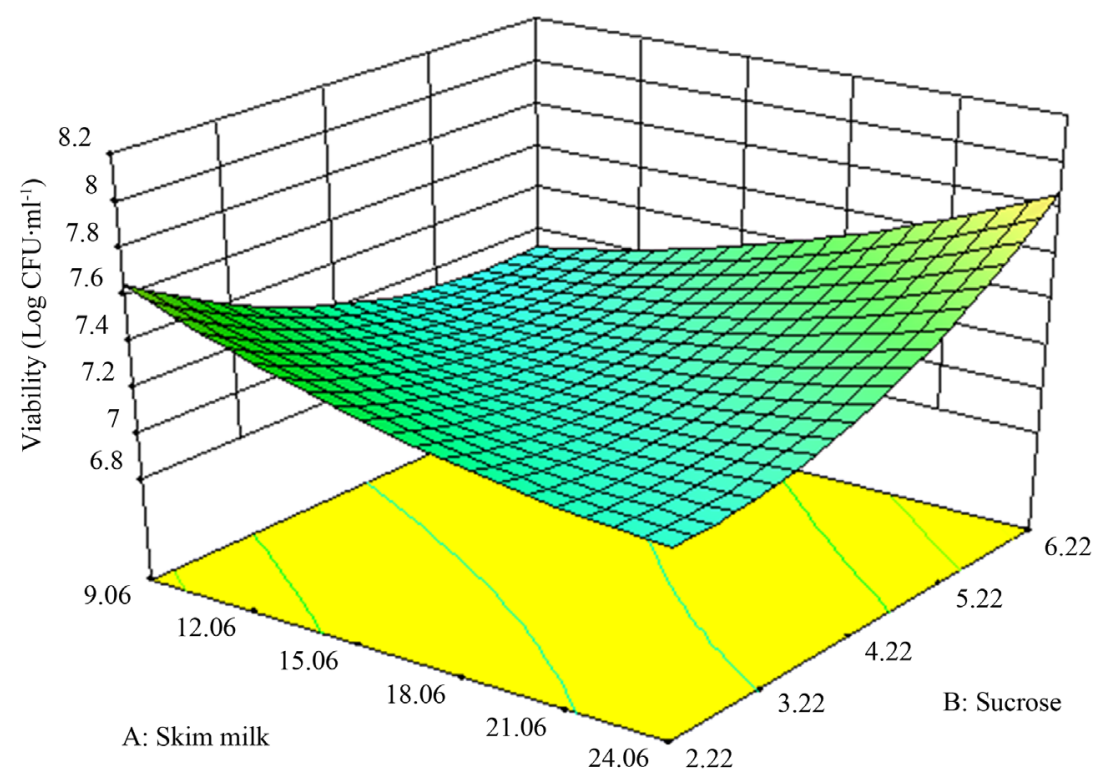

Figure 1. Graphical response surface 3D interaction: skim milk vs. sucrose.

Table 5. Estimated coefficients for the different terms of the polynomial equation.

\begin{tabular}{ccc}
\hline Factor & Estimated coefficient & Standard error \\
\hline Intercept & 7.18 & 0.060 \\
Skim milk & 0.062 & 0.040 \\
Sucrose & 0.042 & 0.040 \\
Trehalose & 0.12 & 0.040 \\
Skim milk x Sucrose & 0.31 & 0.052 \\
Skim milk x Trehalose & -0.11 & 0.052 \\
Sucrose x Trehalose & 0.20 & 0.052 \\
Skim milk $^{2}$ & 0.098 & 0.039 \\
Sucrose $^{2}$ & 0.18 & 0.039 \\
Trehalose $^{2}$ & 0.044 & 0.039 \\
\hline
\end{tabular}

Skim milk and trehalose showed a kind of antagonistic behavior. A decrease in viability was observed by increasing or reducing the concentration of the two components simultaneously, whereas if only one did, viability looked favored. However, these effects were not significant (Figure 2).

In the latter case, we analyzed the interaction between sucrose and trehalose. It was observed a synergistic effect between the two cryoprotectants, which helps increase the viability of the two components of the total premix. The effects were significant (Figure 3).

\section{Discussion}

Skim milk powder, sucrose and trehalose play an important role in preserving the viability of Lactobacillus plantarum when it is subjected to a freeze drying process and storage at room temperature, allowing its conservation and its incorporation into a powder in food premixes for snails in order to incorporate it as a probiotic.

Although it has shown a protective effect on the microorganism tested by the set of cryoconservants in the lyophilization process, it is unclear what their individual effects are. From the results it follows that there is a standardization problem in the preparation of vials. 


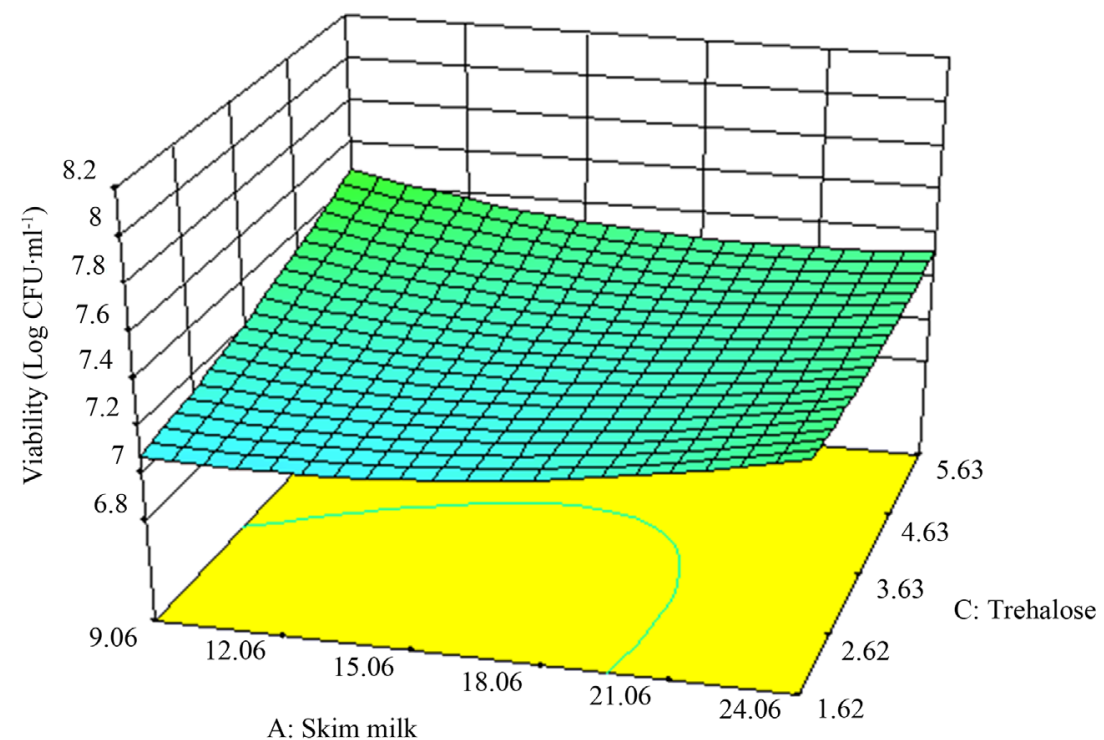

Figure 2. Graphical response surface 3D interaction: skim milk vs. trehalose.

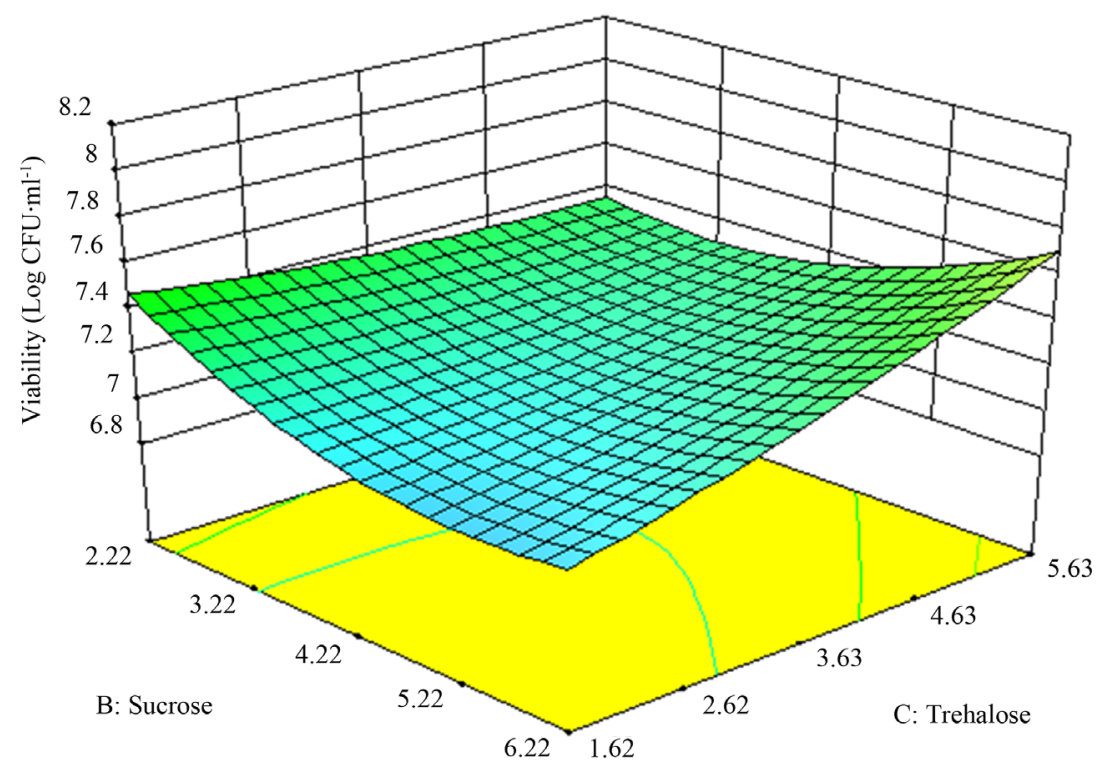

Figure 3. Graphical response surface 3D interaction: sucrose vs trehalose.

The optimal combination of cryoprotectants from the premix obtained by Response Surface Methodology applied to a set of premixes viabilities are determined by the quadratic polynomial equation

$$
Y=7.18+0.062 x_{1}+0.042 x_{2}+0.12 x_{3}+0.31 x_{1} x_{2}+0.11 x_{1} x_{3}+0.20 x_{2} x_{3}+0.098 x_{1}^{2}+0.18 x_{2}^{2}+0.044 x_{3}^{2}
$$

containing 22 possible resolutions, among which is the following composition: $24.06 \%$ skim milk, $6.22 \%$ sucrose, 5.63\% trehalose, which gives an expected 8.12 viability $\left(\log \mathrm{CFU} \cdot \mathrm{mL}^{-1}\right)$.

This analysis also reveals that trehalose has a direct effect on the viability of the premix and skimmed milk and sucrose have quadratic effects on cell viability. There are also interactions between cryoprotectants, which include skim milk-sucrose and sucrose-trehalose synergies, to help maintain the viability of L. plantarum.

\section{References}

[1] Parkes, G.C., Sanderson, J.D. and Whelan, K. (2009) The Mechanisms and Efficacy of Probiotics in the Prevention of 
Clostridium difficile-Associated Diarrhea. The Lancet Infectious Diseases, 9, 237-244. http://dx.doi.org/10.1016/S1473-3099(09)70059-3

[2] Collado, M.C., Isolauri, E., Salminen, S. and Sanz, Y. (2009) The Impact of Probiotic on Gut Health. Current Drug Metabolism, 10, 68-78. http://dx.doi.org/10.2174/138920009787048437

[3] Lomax, A.R. and Calder, P.C. (2009) Probiotics, Immune Function, Infection and Inflammation: A Review of the Evidence from Studies Conducted in Humans. Current Pharmaceutical Design, 15, 1428-1518. http://dx.doi.org/10.2174/138161209788168155

[4] Veerappan, G.R., Betteridge, J. and Young, P.E. (2012) Probiotics for the Treatment of Inflammatory Bowel Disease. Current Gastroenterology Reports, 14, 324-333. http://dx.doi.org/10.1007/s11894-012-0265-5

[5] Vilà, B., Fontgibell, A., Badiola, I., Esteve-Garcia, E., Jiménez, G., Castillo, M. and Brufau, J. (2009) Reduction of Salmonella enterica var. enteritidis Colonization and Invasion by Bacillus cereus var. toyoi Inclusion in Poultry Feeds. Poultry Science, 88, 975-979. http://dx.doi.org/10.3382/ps.2008-00483

[6] Pătruică, S. and Mot, D. (2012) The Effect of Using Prebiotic and Probiotic Products on Intestinal Micro-Flora of the Honeybee (Apis mellifera carpatica). Bulletin of Entomological Research, 102, 619-623. http://dx.doi.org/10.1017/S0007485312000144

[7] Cebrián, R., Baños, A., Valdivia, E., Pérez-Pulido, R., Martínez-Bueno, M. and Maqueda, M. (2012) Characterization of Functional, Safety, and Probiotic Properties of Enterococcus faecalis UGRA10, a New AS-48-Producer Strain. Food Microbiology, 30, 59-67. http://dx.doi.org/10.1016/j.fm.2011.12.002

[8] Huyghebaert, G., Ducatelle, R. and Van Immerseel, F. (2011) An Update on Alternatives to Antimicrobial Growth Promoters for Broilers. The Veterinary Journal, 187, 182-188. http://dx.doi.org/10.1016/j.tvjl.2010.03.003

[9] Nagpal, R., Kumar, A., Kumar, M., Behare, P.V., Jain, S. and Yadav, H. (2012) Probiotics, Their Health Benefits and Applications for Developing Healthier Foods: A Review. FEMS Microbiology Letters, 334, 1-15. http://dx.doi.org/10.1111/j.1574-6968.2012.02593.x

[10] Wright, S. (2009) Homogeneity and Stability of Nutritional Premixes. www.naturalproductsinsider.com/articles/2009/07/homogeneity-and-stability-of-nutritional-premixes.aspx

[11] Carvalho, A.S., Silva, J., Ho, P., Teixeira, P., Malcata, F.X. and Gibbs, P. (2003) Impedimetric Method for Estimating the Residual Activity of Freeze-Dried Lactobacillus delbrueckii ssp. bulgaricus. International Dairy Journal, 13, 463468. http://dx.doi.org/10.1016/S0958-6946(03)00049-9

[12] Li, H.P., Lu, M.J., Guo, H.F., Li, W. and Zhang, H.P. (2010) Protective Effect of Sucrose on the Membrane Properties of Lactobacillus casei Zhang Subjected to Freeze-Drying. Journal of Food Protection, 73, 715-719.

[13] Selmer-Olsen, E., Birkeland, S.E. and Sørhaug, T. (1999) Effect of Protective Solutes on Leakage from and Survival of Immobilized Lactobacillus Subjected to Drying, Storage and Rehydration. Journal of Applied Microbiology, 87, 429437. http://dx.doi.org/10.1046/j.1365-2672.1999.00839.x

[14] Carvalho, A.S., Silva, J., Ho, P., Teixeira, P., Malcata, F.X. and Gibbs, P. (2004) Relevant Factors for the Preparation of Freeze-Dried Lactic Acid Bacteria. International Dairy Journal, 14, 835-847. http://dx.doi.org/10.1016/j.idairyj.2004.02.001

[15] Heckly, R.J. (1985) Principles of Preserving Bacteria by Freeze-Drying. Developments in Industrial Microbiology, 26, 379-395.

[16] Palmfeldt, J., Adsträom, P.R. and Hahn-Häagerdal, B. (2003) Optimisation of Initial Cell Concentration Enhances Freeze-Drying Tolerance of Pseudomonas chlororaphis. Cryobiology, 47, 21-29.

http://dx.doi.org/10.1016/S0011-2240(03)00065-8

[17] FEFANA (2005) Probiotics in Animal Nutrition. www.fefana.org/resources/documents/publications/total\%20def\%20probio.pdf

[18] Font, G., Savoy, G., Pesce, A. and Oliver, G. (1983) Comparative Study of the Efficiency of Some Additives in Protecting Lactic Acid Bacteria against Freeze-Drying. Cryobiology, 20, 560-566. http://dx.doi.org/10.1016/0011-2240(83)90044-5

[19] Schwab, C., Vogel, R. and Gänzle, M.G. (2007) Influence of Oligosaccharides on the Viability and Membrane Properties of Lactobacillus reuteri TMW1.106 during Freeze-Drying. Cryobiology, 55, 108-114. http://dx.doi.org/10.1016/j.cryobiol.2007.06.004

[20] Crow, J.H., Carpenter, J.F. and Crowe, L.M. (1973) The Role of Vitrification in Anhydrobiosis. Annual Review of Physiology, 60, 73-103. http://dx.doi.org/10.1146/annurev.physiol.60.1.73

[21] Villarreal, M.A., Díaz, S.B., Disalvo, E.A. and Montich, G.G. (2004) Molecular Dynamics Simulation Study of the Interaction of Trehalose with Lipid Membranes. Langmuir, 20, 7844-7851. http://dx.doi.org/10.1021/la049485l

[22] Hubalek, Z. (2003) Protectants Used in the Cryopreservation of Microorganisms. Cryobiology, 46, 205-229. 
http://dx.doi.org/10.1016/S0011-2240(03)00046-4

[23] Guilio, B.P., Orlando, P., Barba, G., Coppola, R., De Rosa, M., Sada, A., De Prisco, P. and Nazarro, F. (2005) Use of Alginate and Cryoprotective Sugars to Improve the Viability of Lactic Acid Bacteria after Freezing and Freeze-Drying. World Journal of Microbiology and Biotechnology, 21, 739-746. http://dx.doi.org/10.1007/s11274-004-4735-2

[24] Huang, L., Lu, Z., Yuan, Y., Lü, F. and Bie, X. (2006) Optimization of a Protective Medium for Enhancing the Viability of Freeze-Dried Lactobacillus delbrueckii subsp. bulgaricus Based on Response Surface Methodology. Journal of Industrial Microbiology \& Biotechnology, 33, 55-61. http://dx.doi.org/10.1007/s10295-005-0041-8

[25] Khoramnia, A., Abdullah, N., Liew, S.L., Sieo, C.C., Ramasamy, K. and Ho, Y.W. (2011) Enhancement of Viability of a Probiotic Lactobacillus Strain for Poultry during Freeze-Drying and Storage Using the Response Surface Methodology. Journal of Animal Science, 82, 127-135. http://dx.doi.org/10.1111/j.1740-0929.2010.00804.X

[26] Pérez, L. (2011) Evaluación de nuevas cepas probióticas: Efectos sobre micotoxinas y en Helicicultura. Ph.D. Dissertation, Autonomous University of Barcelona, Bellaterra. 
Scientific Research Publishing (SCIRP) is one of the largest Open Access journal publishers. It is currently publishing more than 200 open access, online, peer-reviewed journals covering a wide range of academic disciplines. SCIRP serves the worldwide academic communities and contributes to the progress and application of science with its publication.

Other selected journals from SCIRP are listed as below. Submit your manuscript to us via either submit@scirp.org or Online Submission Portal.
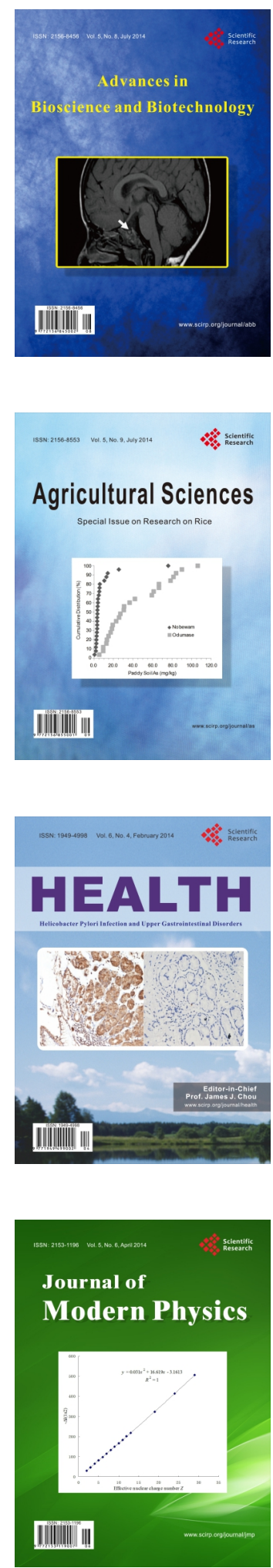
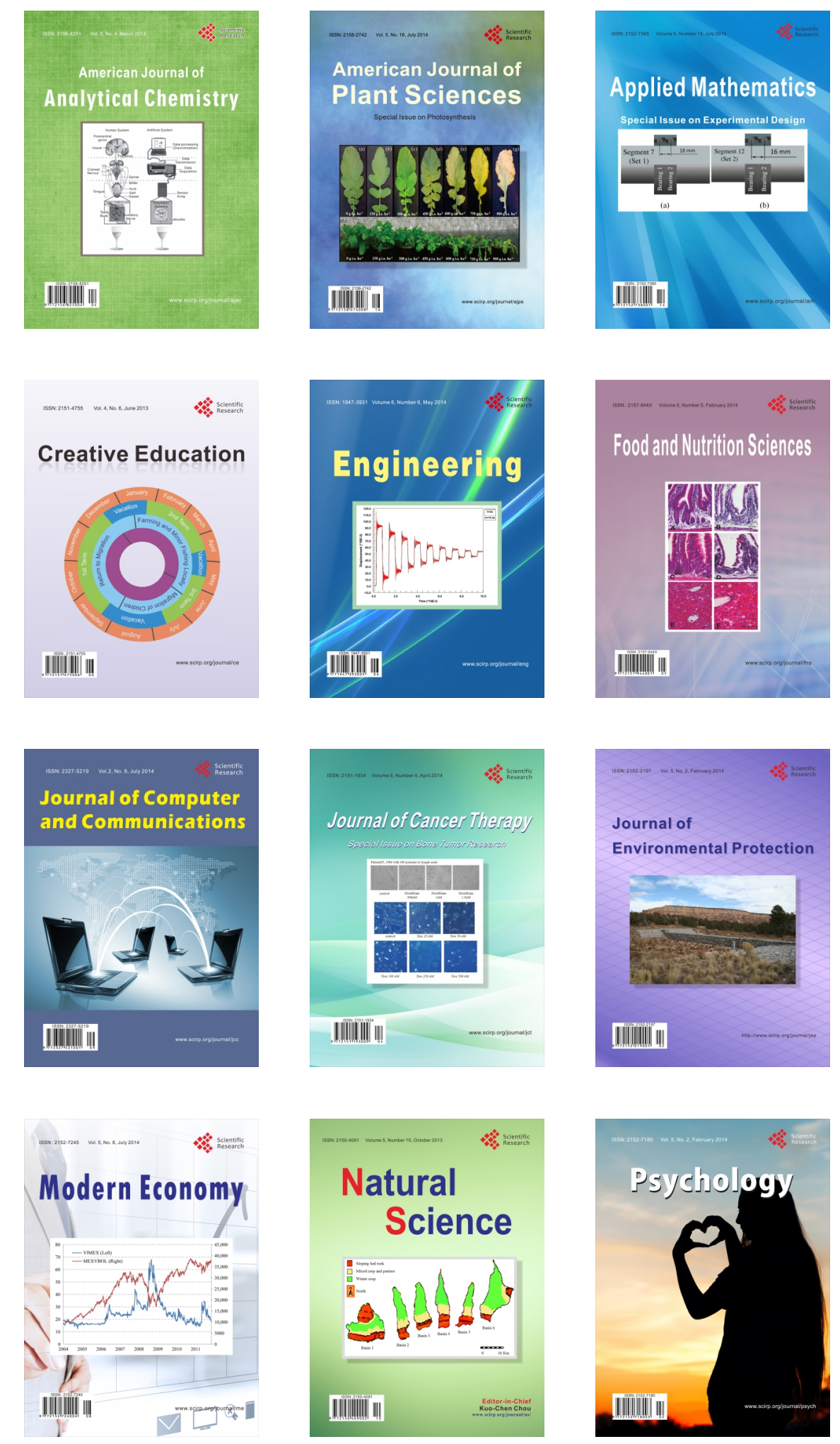